Review Article

\title{
A Review of Surgical Outcomes and Advances for Macular Holes
}

\author{
Peng-peng Zhao $\mathbb{D}^{1},{ }^{1}$ Shuang Wang, ${ }^{2}$ Nan Liu, ${ }^{1}$ Zhi-min Shu, ${ }^{1}$ and Jin-song Zhao $\mathbb{D}^{1}$ \\ ${ }^{1}$ Department of Ophthalmology, The Second Hospital of Jilin University, Changchun, China \\ ${ }^{2}$ Department of Ophthalmology, The Third People's Hospital of Chengdu, Chengdu, China \\ Correspondence should be addressed to Jin-song Zhao; jinsongzhao2003@163.com
}

Received 23 November 2017; Revised 28 January 2018; Accepted 18 February 2018; Published 18 April 2018

Academic Editor: Toshihide Kurihara

Copyright (c) 2018 Peng-peng Zhao et al. This is an open access article distributed under the Creative Commons Attribution License, which permits unrestricted use, distribution, and reproduction in any medium, provided the original work is properly cited.

\begin{abstract}
The surgical outcomes of macular holes (MHs) have improved greatly in recent years. The closure rate is as high as $90-100 \%$, but the outcomes of some special types of MHs remain unsatisfactory. Internal limiting membrane (ILM) peeling dramatically improves the anatomic success rate, but recent studies have found that it could also cause mechanical and subclinical traumatic changes to the retina. Dyes are widely used, and apart from indocyanine green (ICG), the toxicities of other dyes require further research. Facedown posturing is necessary for MHs larger than $400 \mu \mathrm{m}$, and the duration of this posture is determined by the type of tamponade and the case. The ellipsoid zone has been shown to be highly correlated with visual outcome and recovery. New surgical methods include the inverted ILM flap technique and the ILM abrasion technique. However, they require further research to determine their effectiveness.
\end{abstract}

\section{Introduction}

A macular hole $(\mathrm{MH})$ is a full-thickness or partial-thickness defect in the macular region, and its pathogenesis can be idiopathic or result from myopia, trauma, or other causes $[1,2]$. Before the application of vitrectomy, there was no specific treatment for $\mathrm{MH}$ [3], although some $\mathrm{MHs}$ were known to close spontaneously [4]. Surgery for MH has undergone great developments since Kelly and Wendel [5] first applied vitrectomy to treat $\mathrm{MH}$. Both closure rate and visual recovery have improved dramatically; internal limiting membrane (ILM) peeling in particular has significantly improved the closure rate. The use of dyes and the development of microincision surgery have reduced both the duration of surgery and the risk of damage from surgery. Furthermore, a recent postoperative posturing study reported pain relief for patients, and new surgical methods may soon offer novel solutions to the remaining problems. This article reviews the outcomes of and advances in $\mathrm{MH}$ surgery.

\section{The Surgical Outcomes}

2.1. Visual Outcomes. Kelly and Wendel [5] reported that $73 \%$ patients who underwent vitrectomy resulting in successful macular reattachment experienced an improvement in visual acuity of two lines or better. With the continuous improvement in surgical techniques, the closure rate is improved and the rate of visual acuity recovery has been improved.

However, visual acuity outcomes differ by the MH type. Compared with the idiopathic $\mathrm{MH}$, the postoperative visual outcomes for high myopic $\mathrm{MH}$ are limited. For idiopathic MHs, Tewari et al. [6] reported a final visual acuity of 20/50 following ILM peeling. Wu and Kung [4] reported that the mean $\operatorname{logMAR}$ visual acuity improved in a group with high myopia from 0.92 to 0.63 , while a group without high myopia showed an improvement from 1.02 to 0.48 . Thus, visual outcomes were less successful in highly myopic eyes. Due to the relatively less favorable outcomes of ILM peeling techniques for myopic eyes and for large and refractory $\mathrm{MHs}$, the inverted flap technique 
was proposed in 2010. Guber et al. [7] reported that most patients with large MHs (diameter $>400 \mu \mathrm{m}$ ) showed bestcorrected visual acuity (BCVA) improvements of 1 to 2 lines following surgery using the inverted flap technique. Khodani et al. [8] reported that visual acuity was improved in patients with very large $\mathrm{MHs}$ (diameter $>1000 \mu \mathrm{m}$ ), from a baseline visual acuity of $20 / 120$ to a final visual acuity of $20 / 80$ following surgery using the inverted flap technique.

Visual acuity improvements are known to differ depending on the stage of $\mathrm{MH}$ and the type of stain used in ILM peeling. Mean visual acuity has been reported to improve to 20/50 for stage 2 holes, 20/110 for stage 3 holes, and 20/145 for stage 4 holes [9]. Further, better visual outcomes following ILM peeling have been reported with brilliant blue (BB) compared to indocyanine green (ICG). Williamson and Lee [9] reported that postoperative visual acuity was $20 / 100$ for patients who underwent surgery where ICG was used, while the postoperative visual acuity was 20/70 for patients who underwent surgery with $\mathrm{BB}$.

2.2. Closure Rate. Before the introduction of vitrectomy to treat $\mathrm{MH}$, the spontaneous closure rate for Gass stage 3 and $4 \mathrm{MHs}$ was merely $4 \%$, while that for stage $2 \mathrm{MHs}$ was $11.4 \%$ [4]. Following the introduction of vitrectomy by Kelly and Wendel [5], the closure rate increased to $58 \%$. As surgical techniques and instrumentation have improved, the closure rate has increased to as high as $90 \%$ [10].

However, as with visual outcomes, surgical outcomes differ by the MH type. Idiopathic MHs have the best outcomes, with reported closure rates ranging from 90 to $100 \%$ $[6,11-20]$. Vitrectomy can reduce tangential traction at the prefoveal vitreous cortex and/or the epiretinal membranes (ERMs) as well as anteroposterior traction at the vitreoretinal interface. Compared with idiopathic MHs, the postoperative closure rates for myopic, traumatic, and large MHs are limited and these MHs require a second surgery [4, 21-29]. Traumatic $\mathrm{MH}$ is hypothesized to result from axial compression of the globe, which can suddenly reduce the globe's anterior-posterior diameter and cause the eyeball to expand in the equatorial direction to compensate. This change can lead to splitting of the retinal layers at the fovea [21]. The postoperative closure rate for a traumatic $\mathrm{MH}$ has been reported to range from approximately $83 \%$ to $92.3 \%[21,30]$. For a myopic $\mathrm{MH}$, the closure rate has been reported to range from $63 \%$ to $90 \%$ [4, 22-25]. Although the reasons for these less favorable outcomes are not fully understood, some authors speculated that a long axial length $(>30 \mathrm{~mm})$ and posterior staphyloma, which can exert additional traction on the retinal surface and impede hole closure, are the two main causes for the low closure rates and limited visual recovery $[4,26]$.

Additionally, $\mathrm{MH}$ diameter is also an important issue in hole closure $[28,29,31-35]$. One study in particular indicated that when the MHs with diameters less than $400 \mu \mathrm{m}$, the closure rate is approximately $92-97 \%$, while MHs with diameters greater than $500 \mu \mathrm{m}$ show a closure rate of just $50 \%$ [31]. This difference in closure rates is caused by both hole diameter and the associated Gass stage. Indeed, other studies have shown that closure rate differs by stage in idiopathic $\mathrm{MH}$, with lower rates being observed in stage 3 and $4 \mathrm{MHs}$ compared to stage $2 \mathrm{MHs}[9,36-38]$. Williamson and Lee [9] reported that among 351 cases, the stage 2, 3, and 4 closure rates were $95.8 \%, 73.0 \%$, and $56.3 \%$, respectively, and this difference was significant.

2.3. Microstructural Changes. Using histology results, postoperative $\mathrm{MH}$ can be divided into four types: U-shaped closures, V-shaped closures, irregular closures, and flat/open closures [39, 40]. Michalewska et al. [39] described a Ushaped closure as a normal foveal contour that results in the best visual outcomes and is present in about $45 \%$ of all patients. A V-shaped closure, described as a steep foveal contour, has been reported to be present in about $26 \%$ of cases and is associated with less favorable visual outcomes compared to U-shaped closures. Irregular closures show an irregular foveal contours and are reported to be present in about $8.8 \%$ of cases. Flat/open closures are observed in approximately $19 \%$ of cases and show foveal defects of the neurosensory retina with a flattened cuff of fluid around the hole. Visual recovery for flat/open closures is limited.

Before the advent of spectral-domain optical coherence tomography (SD-OCT), authors using time-domain OCT (TD-OCT) found that photoreceptor defects were correlated with postoperative BCVA. However, they could not determine the causing mechanisms and could only describe the foveal contour as irregular or regular due to the limited resolution of TD-OCT [41-43]. SD-OCT has a higher resolution and can enhance the intraretinal architectural morphology, especially that of the photoreceptor layer [41]. There are four distinct hyperreflective lines that can be viewed by SD-OCT: the photoreceptor inner segment/outer segment (IS/OS) junction, the external limiting membrane (ELM), the cone outer segment tips (COST), and the retinal pigment epithelium (RPE) [44]. The International Nomenclature OCT Consensus refers to the IS/OS junction as the ellipsoid zone and the COST as the interdigitation zone [45], and thus we use this nomenclature in the remainder of this review. Studies have reported that defects in the ellipsoid zone are a major reason for unsatisfactory visual recovery, and the length of the ellipsoid zone defect is negatively correlated with visual recovery. Further, as the ellipsoid zone is restored, visual acuity is expected to improve [46-51]. Other studies have found that the ELM and the interdigitation zone are also associated with visual recovery [49]. The ELM has further been shown to be correlated with the ellipsoid zone: a disrupted ELM has been reported to always be accompanied by a disrupted ellipsoid zone, but a restored or intact ELM is not always associated with an intact ellipsoid zone [50]. It has also been demonstrated that the integrity of the ELM and ellipsoid zone is the most important factor related to postoperative visual acuity [44].

A less common mechanism of $\mathrm{MH}$ formation is by ERM traction, usually on a lamellar macular hole (LMH) [52]. Recently, a study proposed by Pang and associates [53] found that the lamellar hole-associated epiretinal proliferation (LHEP) appears on SD-OCT images as a substantial material of homogenous medium reflectivity located on the epiretinal surface, which is a unique feature in LMH or LMH-induced 
full-thickness macular hole (FTMH). In addition, unlike the common configuration observed with $\mathrm{MH}$, SD-OCT images show ERM-induced MHs as narrow base with wider separation in the inner retina [52].

\section{The Surgical Techniques}

3.1. ILM Peeling and New Surgical Methods. Several metaanalyses have indicated that ILM peeling can significantly improve initial postoperative closure rate and visual recovery and reduce the chance of a second operation [54-56]. Several studies have reported that there is no difference between peeling and nonpeeling for stage 1 and $2 \mathrm{MHs}$ but there is a significant difference for stages 3 and 4 between peeling and nonpeeling, regarding the closure rate. ILM peeling releases traction caused by glial cells, which then migrate on the surface of the ILM, as it acts as a scaffold for cellular proliferation $[20,57,58]$. ILM peeling is now a standard procedure in $\mathrm{MH}$ surgery.

Although ILM peeling can result in improvements in $\mathrm{MH}$ treatment, recent research has found that it can cause mechanical and subclinical traumatic changes to the retinal nerve fiber layer (RNFL) [59, 60]. The earliest change is swelling of the arcuate retinal nerve fiber layer (SANFL) [61]. Indeed, Clark et al. [61] found that patients who underwent vitrectomy and ILM peeling presented with hypoautofluorescent arcuate striae in the macular region on infrared and autofluorescence imaging with corresponding hyperreflectantive swelling demonstrated on spectraldomain optical coherence tomography (SD-OCT). The SANFL does not appear to impact the final BCVA and can be expected to disappear in about 3 months [59]. There are two hypotheses regarding the cause of SANFL [61]. The first hypothesis is that surgical forceps cause direct damage to the retina when grasping the ILM while the second is that ILM peeling causes damage to the Müller cell endplates that are attached to the ILM.

The dissociated optic nerve fiber layer (DONFL), which is similar to the SANFL $[10,58,59,62]$, is observed as small, spindle-shaped splitting adjacent nerve fiber bundles on SDOCT. Not all patients who undergo ILM peeling will present with the DONFL postoperatively, and there have been no significant differences observed between eyes with and those without the DONFL with respect to BCVA or macular sensitivity. The reason for DONFL presentation is also unclear, although some authors speculated that the DONFL is caused by irregularly distributed Müller cells following ILM peeling in regions that show a higher density of nerve fiber bundles in the RNFL.

Although ILM peeling is generally used for MH surgery, several surgical technique modifications have been studied in recent years. Bae et al. [20] demonstrated that the extent of ILM peeling affects the degree of postoperative metamorphopsia. They divided the radius of ILM removal into two groups of 0.75 and 1.5 disc diameter (DD) and found that a larger extent of ILM removal was related to significantly better postoperative metamorphopsia improvement. There have also been developments of new surgical methods for MHs that are associated with unsatisfactory anatomic outcomes and visual recovery, including large MHs, highly myopic MHs, and traumatic MHs. Ho et al. [63] performed the foveola nonpeeling surgery in early-stage $2 \mathrm{MHs}$. They peeled off a donut-shaped ILM, leaving a $400 \mu \mathrm{m}$ diameter ILM over the foveola. And in this way, they successfully prevented inner retinal damages, maintained the integrity of the foveolar structure, and led to better final visual acuity. In 2010, Michalewska et al. [27] first adopted the inverted ILM flap technique to treat large MHs. The authors of this study did not grasp the ILM completely but left it attached at the edges of the MH. Next, they rolled the ILM to cover the hole and left the ILM's retinal face adjacent to the vitreous cavity. This new method was shown to significantly improve the closure rates of large MHs $(>400 \mu \mathrm{m})$ and to change the flat/open closures into U-shaped or V-shaped closures. Later, the authors applied this method to highly myopic, traumatic, and other refractory $\mathrm{MHs}$ and were again able to achieve higher closure rates and improved visual outcomes $[7,8$, $25,28,64-71]$. However, the mechanism of the ILM flap technique is not yet clear. The results of a study by Kase et al. [70] suggest that glial cells placed on the hole may produce intermediate filaments and provoke tissue remodeling within the MH. Further, Shiode et al. [72] proposed that the ILM functions as a scaffold for the proliferation and migration of Müller cells, allowing the neurotrophic factors and basic fibroblast growth factors that are produced by the Müller cells to contribute to MH closure.

Recent studies have improved on the inverted ILM flap technique: a rolled segment of the peeled ILM into a singlelayered ILM. A single-layered ILM can now be rolled and used to fill the MH [73-75]. Song et al. [73] developed a vitrectomy combined with a Viscoat- (Alcon Laboratories, Fort Worth, TX, USA) assisted single-layered inverted ILM flap technique. The use of Viscoat effectively prevents retroversion of the ILM flap during the fluid-air exchange and minimizes the toxic effect of ICG staining on the RPE. Morizane et al. [64] further developed an autologous transplantation of the ILM for refractory MHs in which the surgeon grasps the ILM flap from the ungrasped area to cover the hole for patients who undergo ILM peeling. Michalewska et al. [76] reported that the temporal inverted ILM flap technique, which involves grasping the ILM from the temporal area, reduced the incidence of the DONFL and SANFL. Chen and Yang [77] reported a technique that uses the autologous anterior or posterior lens capsule flap as a scaffold to plug the MH. Finally, Grewal and Mahmoud [78] introduced a new technique involving the use of the autologous neurosensory retinal free flap for closure of refractory myopic MHs.

Mahajan et al. [79] reported a new ILM abrasion technique for postvitrectomy in which a diamond-dusted membrane scraper is brushed over the macula in the $1 \mathrm{DD}$ area surrounding the $\mathrm{MH}$. This technique achieves similar results as ILM peeling (total, 94\% closure) and achieves a rate of 93.5\% (58/62) closure for Gass stage 3 and 4 holes [79]. They also found that this method would not penetrate the RNFL [80]. Therefore, they believe that the ILM abrasion technique is another option for $\mathrm{MH}$ surgery. More studies are required to determine the effectiveness of both the inverted ILM flap technique and the ILM abrasion technique. 
3.2. Dyes and Adjuvants. Even for experienced surgeons, it is difficult to visualize the ILM during MH surgery. The application of dyes and adjuvants can make $\mathrm{MH}$ surgeries safer, reduce the duration of surgery, and decrease the risk of mechanical trauma to the retina.

Commonly used dyes include indocyanine green (ICG), trypan blue (TB), brilliant blue G (BBG), and acid violet 17 [58]. Adjuvants include triamcinolone acetonide (TA) and blood [58, 81].

Among these dyes, the earliest and most widely used is ICG [10], but studies have demonstrated that ICG can cause toxicity. ICG has an impact on the retinal ganglion cells (RGCs), glial cells, and RPE cells [82, 83]. Two metaanalyses have reported that eyes treated with ICG have poorer visual acuity and field outcomes than those treated with other dyes $[84,85]$. Additionally, the visual field defect present after ILM removal has been shown to further progress after surgery when ICG is used [86]. TB is another widely used dye [10, 81, 87-89], but several in vitro experiments have shown that it causes dose- and time-dependent neurotoxicity on RGCs. However, the RGC toxicity observed for TB has been less than that observed for ICG $[58,90,91]$.

Authors have also reported RPE atrophy following TBassisted ILM peeling $[92,93]$. TA has been shown to be safe and effective compared with ICG, and its usage has thus become common [81, 94-97]. The main side effect of TA is high postoperative intraocular pressure, and one in vitro study has reported that TA is toxic to RPE cells when applied at a normal dose [98]. However, a pig study failed to observe the same RPE atrophy 6 weeks after surgery [95]. Blood can also be used for staining [58], and one report has demonstrated that the use of whole blood before staining the ILM with BBG causes earlier and better visual postoperative rehabilitation [99]. BBG is another safe dye used in ophthalmological surgery [100]. Although ICG and TB injections have both been shown to cause retinal cell degeneration, subretinal injection of BBG had no such effect in a study using a rat model [101]. One in vitro experiment used ICG, TB, TA, and BBG in a rat model and reported that only ICG caused retinal cell dysfunction and structural damage [102]. Further, one electroretinogram and histopathology study used ICG, TA, and BBG in a pig eye model and reported that the cytotoxicity of ICG is significantly higher than that of TA or BBG [95]. Another experiment found that neither BBG nor TA use had a significant effect on postoperative mfERG (multifocal electroretinogram) responses or histology in pig eye $[95,103]$. A meta-analysis further showed that there is no significant difference in the rate of hole closure following MH surgery using BBG versus other dyes, but significantly better recovery of postoperative visual function was present when BBG was used compared to ICG or other dyes [104]. Acid violet 17 is a dye that has been recently introduced for use in $\mathrm{MH}$ surgery and is specific to the ILM. This dye allows clear intraoperative visualization and provides a greater contrast than BBG. Although its safety has been confirmed at concentrations of $0.25 \mathrm{~g} / \mathrm{L}$ and $0.5 \mathrm{~g} / \mathrm{L}$, further studies are required to confirm its long-term safety [58].
Indeed, further study is required to determine the toxicities of each dye, with the exception of ICG, during MH surgery.

3.3. Postoperative Posturing and Tamponade. When Kelly and Wendel [5] first introduced vitrectomy for $\mathrm{MH}$ treatment, they used room air to fill the vitreous cavity and required that the patients stay face-down for one week. Face-down posturing has thus become a standard of MH treatment.

However, face-down posturing is accompanied by great inconvenience, and some patients, especially children and the elderly, cannot tolerate it. Therefore, there is still debate about the necessity and duration of face-down posturing $[105,106]$. A meta-analysis by $\mathrm{Hu}$ and colleagues [107] found that patients who stay face-down show better anatomic outcomes than those who do not. However, when the MH diameter was $<400 \mu \mathrm{m}$, face-down posturing showed no significant difference in anatomic success, while facedown posturing was associated with a higher success rate when the $\mathrm{MH}$ diameter was $>400 \mu \mathrm{m}$ [108]. Thus, facedown posturing currently seems to be necessary, especially for large MHs.

The duration of face-down posturing is strongly related to the type of tamponade that is used in $\mathrm{MH}$ surgery. Two kinds of tamponades can be used in the vitreous cavity: gas and silicone oil. Gas plays a very important role in MH surgery because air not only can provide scaffolds for cellular proliferation but also can cause the extrusion of subretinal fluid from surface tension [109]. Although room air was originally used for $\mathrm{MH}$ surgery, long-lasting gas can also be applied [105]. Then, longlasting gas became widely used to improve the effect of the gas, and the duration was extended to 1 month. Recently, some researchers have found that room air can have similar outcomes to a long-lasting gas, but the hole diameters in their cases were small, so the conclusions that can be drawn are limited $[110,111]$. Commonly used long-lasting gases include sulfur hexafluoride $\left(\mathrm{SF}_{6}\right)$ and perfluoropropane $\left(\mathrm{C}_{3} \mathrm{~F}_{8}\right)$ [112], and no significant differences in anatomic success or visual outcomes have been reported between these $[108,112-114]$. When longlasting gas became widely used as an improved tamponade, the duration was extended to 1 month. Recently, two studies have reported that room air can have similar outcomes to long-lasting gas, but the conclusions that can be drawn from these studies are limited because they only included holes with small diameters $[110,111]$. The degree of gas fill also affects $\mathrm{MH}$ closure, as a gas fill above at least $65 \%$ on postoperative day 4 has been shown to reduce the risk of poor gas-macula contact and surgical failure [106]. Kannan et al. [115] reported that a smaller volume of $\mathrm{SF}_{6}$ can be used for a longer duration in order to achieve good surgical outcomes at a decreased cost.

Silicone oil, which is also used as a tamponade, is available in heavy and light varieties [116]. There is no consensus about which of these is better with respect to closure rate [117], but one report found that high intraocular pressure is more common following the use of heavy silicone oil 
[118]. Heavy silicone oil can also cause complications such as intraocular inflammation reaction, media opacification, and secondary glaucoma $[119,120]$. Face-down posturing is not strictly necessary when using silicone oil $[83,116]$, and heavy silicone oil only requires that the patient be lied flat [116]. Silicone oil is mainly used for patients who cannot tolerate face-down posturing (e.g., children) [121], for large MHs [21], for MHs that remain open after the first operation [122], for highly myopic MHs with retinal detachment, and for cases involving posterior staphyloma [117]. Studies have demonstrated that initial closure rates and visual outcomes are lower with silicone oil than with gas [111, 123-126], mainly because of silicone oil's lower buoyant force [124], toxic effect on photoreceptor cells, and the cases that are chosen for silicone oil use $[21,83,124]$. In addition, the use of silicone oil requires another operation to remove it. Finally, a recent study has indicated that the use of ILM flap repositioning and autologous gluconated blood clumps as a macular plug is effective in achieving satisfactory hole closure with statically significant functional improvements and in reducing the occurrence of cataract and high intraocular pressure after surgery [127].

Apart from the tamponade type, the duration of facedown posturing is also dependent on the $\mathrm{MH}$ itself. One study indicated that most MHs close during the first postoperative day [128]. Research using short-lasting gases and shorter durations has shown similar outcomes [83, 109, 129-131]. Tatham and Banerjee's meta-analysis found that a duration of $24 \mathrm{~h}$ versus 5-10 days resulted in no significant differences in the closure rate [132]. Iezzi and Kapoor [133] reported that MH surgery using broad ILM peeling, 20\% $\mathrm{SF}_{6}$ gas, and no face-down positioning is highly effective for idiopathic $\mathrm{MH}$. More studies are needed to investigate short durations of face-down posturing.

\section{Conclusion}

There is no doubt that MH surgery has made huge progress leading to better accuracy and convenience and less damage. The continual development of new instruments helps surgeons to better assess microstructural changes, while new surgical methods provide a promising direction for treatment. However, these new advances are still being explored, and more research is needed in order to develop more definitive conclusions.

\section{Conflicts of Interest}

The authors declare that there is no conflict of interest regarding the publication of this article.

\section{Acknowledgments}

This study was supported by "Binding activity of sugar glycosaminoglycans and RPE mediated by CD44 receptor" (NO20160101094JC) and "The project of emergency treatment for ocular trauma" (3D5172173429).

\section{References}

[1] M. Landolfi, M. A. Zarbin, and N. Bhagat, "Macular holes," Ophthalmology Clinics of North America, vol. 15, no. 4, pp. 565-572, 2002.

[2] M. la Cour and J. Friis, "Macular holes: classification, epidemiology, natural history and treatment," Acta Ophthalmologica, vol. 80, no. 6, pp. 579-587, 2002.

[3] A. R. Margherio, "Macular hole surgery in 2000," Current Opinion in Ophthalmology, vol. 11, no. 3, pp. 186-190, 2000.

[4] T. T. Wu and Y. H. Kung, "Comparison of anatomical and visual outcomes of macular hole surgery in patients with high myopia vs. non-high myopia: a case-control study using optical coherence tomography," Graefe's Archive for Clinical and Experimental Ophthalmology, vol. 250, no. 3, pp. 327-331, 2012.

[5] N. E. Kelly and R. T. Wendel, "Vitreous surgery for idiopathic macular holes. Results of a pilot study," Archives of Ophthalmology, vol. 109, no. 5, pp. 654-659, 1991.

[6] A. Tewari, A. Almony, and G. K. Shah, "Macular hole closure with triamcinolone-assisted internal limiting membrane peeling," Retina, vol. 28, no. 9, pp. 1276-1279, 2008.

[7] J. Guber, C. Lang, and C. Valmaggia, "Internal limiting membrane flap techniques for the repair of large macular holes: a short-term follow-up of anatomical and functional outcomes," Klinische Monatsblätter für Augenheilkunde, vol. 234, no. 4, pp. 493-496, 2017.

[8] M. Khodani, P. Bansal, R. Narayanan, and J. Chhablani, "Inverted internal limiting membrane flap technique for very large macular hole," International journal of Ophthalmology, vol. 9, no. 8, pp. 1230-1232, 2016.

[9] T. H. Williamson and E. Lee, "Idiopathic macular hole: analysis of visual outcomes and the use of indocyanine green or brilliant blue for internal limiting membrane peel," Graefes Archive for Clinical and Experimental Ophthalmology, vol. 252, no. 3, pp. 395-400, 2014.

[10] E. K. Chin, D. R. P. Almeida, and E. H. Sohn, "Structural and functional changes after macular hole surgery: a review," International Ophthalmology Clinics, vol. 54, no. 2, pp. 17-27, 2014.

[11] L. Jančo, R. Vida, M. Bartoš, and K. Villémová, "Surgical treatment of the idiopatic macular hole - our experience," Ceska A Slovenska Oftalmologie Casopis Ceske Oftalmologicke Spolecnosti A Slovenske Oftalmologicke Spolecnosti, vol. 69, no. 3, pp. 102-105, 2013.

[12] F. Jordan, S. Jentsch, R. Augsten, J. Strobel, and J. Dawczynski, "Study on the time course of macular pigment density measurement in patients with a macular hole - clinical course and impact of surgery," Klinische Monatsbltter Für Augenheilkunde, vol. 229, no. 11, pp. 1124-1129, 2012.

[13] T. Baba, S. Yamamoto, R. Kimoto, T. Oshitari, and E. Sato, "Reduction of thickness of ganglion cell complex after internal limiting membrane peeling during vitrectomy for idiopathic macular hole," Eye, vol. 26, no. 9, pp. 1173-1180, 2012.

[14] H. Sakaguchi, M. Ohji, Y. Oshima et al., "Long-term followup after vitrectomy to treat idiopathic full-thickness macular holes: visual acuity and macular complications," Clinical Ophthalmology, vol. 6, pp. 1281-1286, 2012.

[15] D. R. P. Almeida, J. Wong, M. Belliveau, J. Rayat, and J. Gale, "Anatomical and visual outcomes of macular hole surgery with short-duration 3-day face-down positioning," Retina, vol. 32, no. 3, pp. 506-510, 2012. 
[16] K. Yamamoto and S. Hori, "Long-term outcome of vitrectomy combined with internal limiting membrane peeling for idiopathic macular holes," Nippon Ganka Gakkai Zasshi, vol. 115, no. 1, pp. 20-26, 2011.

[17] H. Nomoto, F. Shiraga, H. Yamaji et al., "Macular hole surgery with triamcinolone acetonide-assisted internal limiting membrane peeling: one-year results," Retina, vol. 28, no. 3, pp. 427-432, 2008.

[18] T. Hikichi, Y. Furukawa, H. Ohtsuka et al., "Improvement of visual acuity one-year after vitreous surgery in eyes with residual triamcinolone acetonide at the macular hole," American Journal of Ophthalmology, vol. 145, no. 2, pp. 267-272.e1, 2008.

[19] K. Kumagai, M. Furukawa, N. Ogino, E. Larson, and A. Uemura, "Long-term outcomes of macular hole surgery with triamcinolone acetonide-assisted internal limiting membrane peeling," Retina, vol. 27, no. 9, pp. 1249-1254, 2007.

[20] K. Bae, S. W. Kang, J. H. Kim, S. J. Kim, J. M. Kim, and J. M. Yoon, "Extent of internal limiting membrane peeling and its impact on macular hole surgery outcomes: a randomized trial," American Journal of Ophthalmology, vol. 169, pp. 179-188, 2016.

[21] J. B. Miller, Y. Yonekawa, D. Eliott, and D. G. Vavvas, “A review of traumatic macular hole: diagnosis and treatment," International Ophthalmology Clinics, vol. 53, no. 4, pp. 59-67, 2013.

[22] M. Alkabes, L. Padilla, C. Salinas et al., "Assessment of OCT measurements as prognostic factors in myopic macular hole surgery without foveoschisis," Graefe's Archive for Clinical and Experimental Ophthalmology, vol. 251, no. 11, pp. 2521-2527, 2013.

[23] K. Suda, M. Hangai, and N. Yoshimura, "Axial length and outcomes of macular hole surgery assessed by spectraldomain optical coherence tomography," American Journal of Ophthalmology, vol. 151, no. 1, pp. 118-127.e1, 2011.

[24] J. Qu, M. Zhao, Y. Jiang, and X. Li, "Vitrectomy outcomes in eyes with high myopic macular hole without retinal detachment," Retina, vol. 32, no. 2, pp. 275-280, 2012.

[25] S. Kuriyama, H. Hayashi, Y. Jingami, N. Kuramoto, J. Akita, and M. Matsumoto, "Efficacy of inverted internal limiting membrane flap technique for the treatment of macular hole in high myopia," American Journal of Ophthalmology, vol. 156, no. 1, pp. 125-131.e1, 2013.

[26] M. Alkabes, F. Pichi, P. Nucci et al., "Anatomical and visual outcomes in high myopic macular hole (HM-MH) without retinal detachment: a review," Graefe's Archive for Clinical and Experimental Ophthalmology, vol. 252, no. 2, pp. 191199, 2014.

[27] Z. Michalewska, J. Michalewski, R. A. Adelman, and J. Nawrocki, "Inverted internal limiting membrane flap technique for large macular holes," Ophthalmology, vol. 117, no. 10, pp. 2018-2025, 2010.

[28] P. Mahalingam and K. Sambhav, "Surgical outcomes of inverted internal limiting membrane flap technique for large macular hole," Indian Journal of Ophthalmology, vol. 61, no. 10, pp. 601-603, 2013.

[29] S. Ullrich, C. Haritoglou, C. Gass, M. Schaumberger, M. W. Ulbig, and A. Kampik, "Macular hole size as a prognostic factor in macular hole surgery," British Journal of Ophthalmology, vol. 86, no. 4, pp. 390-393, 2002.
[30] N. Brennan, I. Reekie, A. P. Khawaja, N. Georgakarakos, and E. Ezra, "Vitrectomy, inner limiting membrane peel, and gas tamponade in the management of traumatic paediatric macular holes: a case series of 13 patients," Ophthalmologica, vol. 238, no. 3, pp. 119-123, 2017.

[31] A. Susini and P. Gastaud, "Macular holes that should not be operated," Journal Français Dophtalmologie, vol. 31, no. 2, pp. 214-220, 2008.

[32] R. Reis, N. Ferreira, and A. Meireles, "Management of stage IV macular holes: when standard surgery fails," Case Reports in Ophthalmology, vol. 3, no. 2, pp. 240-250, 2012.

[33] Z. Michalewska, J. Michalewski, and J. Nawrocki, "Long-term decrease of retinal pigment epithelium defects in large stage IV macular holes with borders mechanically joined during surgery," Case Reports in Ophthalmology, vol. 2, no. 2, pp. 215-221, 2011.

[34] T. Brockmann, C. Steger, M. Weger, A. Wedrich, and A. Haas, "Risk assessment of idiopathic macular holes undergoing vitrectomy with dye-assisted internal limiting membrane peeling," Retina, vol. 33, no. 6, pp. 1132-1136, 2013.

[35] T. M. Jenisch, F. Zeman, M. Koller, D. A. Märker, H. Helbig, and W. A. Herrmann, "Macular hole surgery: an analysis of risk factors for the anatomical and functional outcomes with a special emphasis on the experience of the surgeon," Clinical Ophthalmology, vol. 11, pp. 1127-1134, 2017.

[36] A. K. Kwok, T. Y. Lai, W. Man-Chan, and D. C. Woo, "Indocyanine green assisted retinal internal limiting membrane removal in stage 3 or 4 macular hole surgery," British Journal of Ophthalmology, vol. 87, no. 1, pp. 71-74, 2003.

[37] S. Foulquier, A. Glacet-Bernard, M. Sterkers, G. Soubrane, and G. Coscas, "Study of internal limiting membrane peeling in stage-3 and -4 idiopathic macular hole surgery," Journal Français Dophtalmologie, vol. 25, no. 10, pp. 1026-1031, 2002.

[38] S. W. Kang, K. Ahn, and D. I. Ham, "Types of macular hole closure and their clinical implications," British Journal of Ophthalmology, vol. 87, no. 8, pp. 1015-1019, 2003.

[39] Z. Michalewska, J. Michalewski, S. Cisiecki, R. Adelman, and J. Nawrocki, "Correlation between foveal structure and visual outcome following macular hole surgery: a spectral optical coherence tomography study," Graefe's Archive for Clinical and Experimental Ophthalmology, vol. 246, no. 6, pp. 823$830,2008$.

[40] M. Imai, H. Iijima, T. Gotoh, and S. Tsukahara, "Optical coherence tomography of successfully repaired idiopathic macular holes," American Journal of Ophthalmology, vol. 128, no. 5, pp. 621-627, 1999.

[41] M. Inoue, Y. Watanabe, A. Arakawa, S. Sato, S. Kobayashi, and K. Kadonosono, "Spectral-domain optical coherence tomography images of inner/outer segment junctions and macular hole surgery outcomes," Graefes Archive for Clinical and Experimental Ophthalmology, vol. 247, no. 3, pp. 325330, 2009.

[42] T. Baba, S. Yamamoto, M. Arai et al., "Correlation of visual recovery and presence of photoreceptor inner/outer segment junction in optical coherence images after successful macular hole repair," Retina, vol. 28, no. 3, pp. 453-458, 2008.

[43] C. Haritoglou, A. S. Neubauer, I. W. Reiniger, S. G. Priglinger, C. A. Gass, and A. Kampik, "Long-term functional outcome of macular hole surgery correlated to optical coherence tomography measurements," Clinical \& Experimental Ophthalmology, vol. 35, no. 3, pp. 208-213, 2007. 
[44] G. Landa, R. C. Gentile, P. M. T. Garcia, T. O. Muldoon, and R. B. Rosen, "External limiting membrane and visual outcome in macular hole repair: spectral domain OCT analysis," Eye, vol. 26, no. 1, pp. 61-69, 2012.

[45] G. Staurenghi, S. Sadda, U. Chakravarthy, R. F. Spaide, and International Nomenclature for Optical Coherence Tomography (IN•OCT) Panel, "Proposed lexicon for anatomic landmarks in normal posterior segment spectral-domain optical coherence tomography: the IN•OCT consensus," Ophthalmology, vol. 121, no. 8, pp. 1572-1578, 2014.

[46] M. Sano, Y. Shimoda, H. Hashimoto, and S. Kishi, "Restored photoreceptor outer segment and visual recovery after macular hole closure," American Journal of Ophthalmology, vol. 147, no. 2, pp. 313-318, 2009.

[47] M. Shimozono, A. Oishi, M. Hata, and Y. Kurimoto, "Restoration of the photoreceptor outer segment and visual outcomes after macular hole closure: spectral-domain optical coherence tomography analysis," Graefe's Archive for Clinical and Experimental Ophthalmology, vol. 249, no. 10, pp. 14691476,2011

[48] J. Oh, W. E. Smiddy, H. W. Flynn Jr, G. Gregori, and B. Lujan, "Photoreceptor inner/outer segment defect imaging by spectral domain OCT and visual prognosis after macular hole surgery," Investigative Ophthalmology \& Visual Science, vol. 51, no. 3, pp. 1651-1658, 2010.

[49] J. M. Ruiz-Moreno, F. Lugo, J. A. Montero, and D. P. Piñero, "Restoration of macular structure as the determining factor for macular hole surgery outcome," Graefe's Archive for Clinical and Experimental Ophthalmology, vol. 250, no. 10, pp. 1409-1414, 2012.

[50] Y. Mitamura, S. Mitamura-Aizawa, T. Katome et al., "Photoreceptor impairment and restoration on optical coherence tomographic image," Journal of Ophthalmology, vol. 2013, Article ID 518170, 7 pages, 2013.

[51] I. Y. Wong, L. P. Iu, H. Koizumi, and W. W. Lai, "The inner segment/outer segment junction: what have we learnt so far?," Current Opinion in Ophthalmology, vol. 23, no. 3, pp. 210-218, 2012.

[52] C. Y. Tsai, Y. T. Hsieh, and C. M. Yang, "Epiretinal membrane-induced full-thickness macular holes: the clinical features and surgical outcomes," Retina, vol. 36, no. 9, pp. 1679-1687, 2016.

[53] C. E. Pang, R. F. Spaide, and K. B. Freund, "Epiretinal proliferation seen in association with lamellar macular holes: a distinct clinical entity," Retina, vol. 34, no. 8, pp. 1513-1523, 2014.

[54] V. Mester and F. Kuhn, "Internal limiting membrane removal in the management of full-thickness macular holes," American Journal of Ophthalmology, vol. 129, no. 6, pp. 769$777,2000$.

[55] D. Tognetto, R. Grandin, G. Sanguinetti et al., "Internal limiting membrane removal during macular hole surgery: results of a multicenter retrospective study," Ophthalmology, vol. 113, no. 8, pp. 1401-1410, 2006.

[56] K. Spiteri Cornish, N. Lois, N. Scott et al., "Vitrectomy with internal limiting membrane (ILM) peeling versus vitrectomy with no peeling for idiopathic full-thickness macular hole (FTMH)," Cochrane Database of Systematic Reviews, vol. 5, no. 6, 2013.

[57] A. Almony, E. Nudleman, G. K. Shah et al., "Techniques, rationale, and outcomes of internal limiting membrane peeling," Retina, vol. 32, no. 5, pp. 877-891, 2012.
[58] F. Morescalchi, C. Costagliola, E. Gambicorti, S. Duse, M. R. Romano, and F. Semeraro, "Controversies over the role of internal limiting membrane peeling during vitrectomy in macular hole surgery," Survey of Ophthalmology, vol. 62, no. 1, pp. 58-69, 2016.

[59] F. Pichi, A. Lembo, M. Morara et al., "Early and late inner retinal changes after inner limiting membrane peeling," International Ophthalmology, vol. 34, no. 2, pp. 437-446, 2014.

[60] A. Modi, A. Giridhar, and M. Gopalakrishnan, "Comparative analysis of outcomes with variable diameter internal limiting membrane peeling in surgery for idiopathic macular hole repair," Retina, vol. 37, no. 2, pp. 265-273, 2016.

[61] A. Clark, N. Balducci, F. Pichi et al., "Swelling of the arcuate nerve fiber layer after internal limiting membrane peeling," Retina, vol. 32, no. 8, pp. 1608-1613, 2012.

[62] R. F. Spaide, “"Dissociated optic nerve fiber layer appearance” after internal limiting membrane removal is inner retinal dimpling," Retina, vol. 32, no. 9, pp. 1719-1726, 2012.

[63] T. C. Ho, C. M. Yang, J. S. Huang, C. H. Yang, and M. S. Chen, "Foveola nonpeeling internal limiting membrane surgery to prevent inner retinal damages in early stage 2 idiopathic macula hole," Graefe's Archive for Clinical and Experimental Ophthalmology, vol. 252, no. 10, pp. 15531560, 2014.

[64] Y. Morizane, F. Shiraga, S. Kimura et al., "Autologous transplantation of the internal limiting membrane for refractory macular holes," American Journal of Ophthalmology, vol. 157, no. 4, pp. 861-869.e1, 2014.

[65] Z. Michalewska and J. Nawrocki, "Macular hole surgery in a patient who cannot maintain facedown positioning," Case Reports in Ophthalmology, vol. 4, no. 1, pp. 1-6, 2013.

[66] H. Hayashi and S. Kuriyama, "Foveal microstructure in macular holes surgically closed by inverted internal limiting membrane flap technique," Retina, vol. 34, no. 12, pp. 2444-2450, 2014.

[67] M. Hirano, Y. Morizane, T. Kawata et al., "Case report: successful closure of a large macular hole secondary to uveitis using the inverted internal limiting membrane flap technique," BMC Ophthalmology, vol. 15, no. 83, 2015.

[68] Z. Michalewska, J. Michalewski, K. Dulczewska-Cichecka, and J. Nawrocki, "Inverted internal limiting membrane flap technique for surgical repair of myopic macular holes," Retina, vol. 34, no. 4, pp. 664-669, 2014.

[69] Z. Chen, C. Zhao, J. J. Ye, X. Q. Wang, and R. F. Sui, "Inverted internal limiting membrane flap technique for repair of large macular holes: a short-term follow-up of anatomical and functional outcomes," Chinese Medical Journal, vol. 129, no. 5, pp. 511-517, 2016.

[70] S. Kase, W. Saito, S. Mori et al., "Clinical and histological evaluation of large macular hole surgery using the inverted internal limiting membrane flap technique," Clinical Ophthalmology, vol. 11, pp. 9-14, 2017.

[71] A. Oleñik, J. Rios, and C. Mateo, "Inverted internal limiting membrane flap technique for macular holes in high myopia with axial length $\geq 30 \mathrm{~mm}$," Retina, vol. 36 , no. 9, pp. $1688-$ 1693, 2016.

[72] Y. Shiode, Y. Morizane, R. Matoba et al., "The role of inverted internal limiting membrane flap in macular hole closure," Investigate Ophthalmology \& Visual Science, vol. 58, no. 11, pp. 4847-4855, 2017. 
[73] Z. Song, M. Li, J. Liu, X. Hu, Z. Hu, and D. Chen, "Viscoat assisted inverted internal limiting membrane flap technique for large macular holes associated with high myopia," Journal of Ophthalmology, vol. 2016, Article ID 8283062, 7 pages, 2016.

[74] S. N. Chen, "Large semicircular inverted internal limiting membrane flap in the treatment of macular hole in high myopia," Graefes Archive for Clinical and Experimental Ophthalmology, vol. 255, no. 12, pp. 2337-2345, 2017.

[75] K. Y. Pak, J. Y. Park, S. W. Park, I. S. Byon, and J. E. Lee, "Efficacy of the perfluoro-N-octane-assisted single-layered inverted internal limiting membrane flap technique for large macular holes," Ophthalmologica, vol. 238, no. 3, pp. 133-138, 2017.

[76] Z. Michalewska, J. Michalewski, K. Dulczewska-Cichecka, R. A. Adelman, and J. Nawrocki, "Temporal inverted internal limiting membrane flap technique versus classic inverted internal limiting membrane flap technique: a comparative study," Retina, vol. 35, no. 9, pp. 1844-1850, 2015.

[77] S. N. Chen and C. M. Yang, "Lens capsular flap transplantation in the management of refractory macular hole from multiple etiologies," Retina, vol. 36, no. 1, pp. 163-170, 2016.

[78] D. S. Grewal and T. H. Mahmoud, "Autologous neurosensory retinal free flap for closure of refractory myopic macular holes," JAMA Ophthalmology, vol. 134, no. 2, pp. 229-230, 2016.

[79] V. B. Mahajan, E. K. Chin, R. M. Tarantola et al., "Macular hole closure with internal limiting membrane abrasion technique," JAMA Ophthalmology, vol. 133, no. 6, pp. 635-641, 2015.

[80] D. R. Almeida, E. K. Chin, R. M. Tarantola et al., "Effect of internal limiting membrane abrasion on retinal tissues in macular holes," Investigative Ophthalmology \& Visual Science, vol. 56, no. 5, pp. 2783-2789, 2015.

[81] E. Abdelkader and N. Lois, "Internal limiting membrane peeling in vitreo-retinal surgery," Survey of Ophthalmology, vol. 53, no. 4, pp. 368-396, 2008.

[82] A. Gandorfer, C. Haritoglou, and A. Kampik, "Toxicity of indocyanine green in vitreoretinal surgery," Developments in Ophthalmology, vol. 42, pp. 69-81, 2008.

[83] J. Bainbridge, E. Herbert, and Z. Gregor, "Macular holes: vitreoretinal relationships and surgical approaches," Eye, vol. 22, no. 10, pp. 1301-1309, 2008.

[84] Y. Wu, W. Zhu, D. Xu et al., "Indocyanine green-assisted internal limiting membrane peeling in macular hole surgery: a meta-analysis," PLoS One, vol. 7, no. 11, article e48405, 2012.

[85] E. B. Rodrigues and C. H. Meyer, "Meta-analysis of chromovitrectomy with indocyanine green in macular hole surgery," Ophthalmologica, vol. 222, no. 2, pp. 123-129, 2008.

[86] M. Nakazawa, H. Terasaki, T. Yamashita, A. Uemura, and T. Sakamoto, "Changes in visual field defects during 10-year follow-up for indocyanine green-assisted macular hole surgery," Japanese Journal of Ophthalmology, vol. 60, no. 387, pp. 383-5, 2016.

[87] D. Shukla, J. Kalliath, N. Neelakantan, K. B. Naresh, and K. Ramasamy, "A comparison of brilliant blue G, trypan blue, and indocyanine green dyes to assist internal limiting membrane peeling during macular hole surgery," Retina, vol. 31, no. 10, pp. 2021-2025, 2011.
[88] C. Bellerive, B. Cinq-Mars, M. Louis et al., "Retinal function assessment of trypan blue versus indocyanine green assisted internal limiting membrane peeling during macular hole surgery," Canadian Journal of Ophthalmology, vol. 48, no. 2, pp. 104-109, 2013.

[89] E. A. Abdelkader, M. B. VA, M. Anand, N. W. Scott, M. A. Rehman Siddiqui, and N. Lois, "In vivo safety of trypan blue use in vitreoretinal surgery," Retina, vol. 31, no. 6, pp. 11221127, 2011.

[90] Y. Jin, S. Uchida, Y. Yanagi, M. Aihara, and M. Araie, "Neurotoxic effects of trypan blue on rat retinal ganglion cells," Experimental Eye Research, vol. 81, no. 4, pp. 395400, 2005.

[91] L. Kodjikian, T. Richter, M. Halberstadt et al., "Toxic effects of indocyanine green, infracyanine green, and trypan blue on the human retinal pigmented epithelium," Graefe's Archive for Clinical and Experimental Ophthalmology, vol. 243, no. 9, pp. 917-925, 2005.

[92] S. Jain, K. Kishore, and Y. R. Sharma, "Progressive atrophy of retinal pigment epithelium after trypan-blue-assisted ILM peeling for macular hole surgery," Indian Journal of Ophthalmology, vol. 61, no. 5, pp. 235-237, 2013.

[93] M. U. Saeed and H. Heimann, "Atrophy of the retinal pigment epithelium following vitrectomy with trypan blue," International Ophthalmology, vol. 29, no. 4, pp. 239-241, 2009.

[94] M. S. Tsipursky, M. A. Heller, S. A. de Souza et al., "Comparative evaluation of no dye assistance, indocyanine green and triamcinolone acetonide for internal limiting membrane peeling during macular hole surgery," Retina, vol. 33 , no. 6 , pp. 1123-1131, 2013.

[95] R. Ejstrup, M. la Cour, S. Heegaard, and J. F. Kiilgaard, "Toxicity profiles of subretinal indocyanine green, brilliant blue G, and triamcinolone acetonide: a comparative study," Graefes Archive for Clinical \& Experimental Ophthalmology, vol. 250, no. 5, pp. 669-677, 2012.

[96] S. Taiji and T. Ishibashi, "Visualizing vitreous in vitrectomy by triamcinolone," Graefes Archive for Clinical \& Experimental Ophthalmology, vol. 247, no. 9, pp. 1153-1163, 2009.

[97] S. Machida, Y. Toba, T. Nishimura, T. Ohzeki, K. Murai, and D. Kurosaka, "Comparisons of cone electroretinograms after indocyanine green-, brilliant blue G-, or triamcinolone acetonide-assisted macular hole surgery," Graefe's Archive for Clinical and Experimental Ophthalmology, vol. 252, no. 9, pp. 1423-1433, 2014.

[98] R. Narayanan, J. K. Mungcal, M. C. Kenney, G. M. Seigel, and B. D. Kuppermann, "Toxicity of triamcinolone acetonide on retinal neurosensory and pigment epithelial cells," Investigative Ophthalmology \& Visual Science, vol. 47, no. 2, pp. 722-728, 2006.

[99] B. Ghosh, S. Arora, N. Goel et al., "Comparative evaluation of sequential intraoperative use of whole blood followed by brilliant blue versus conventional brilliant blue staining of internal limiting membrane in macular hole surgery," Retina, vol. 36, no. 8, pp. 1463-1468, 2016.

[100] H. Enaida and T. Ishibashi, "Brilliant blue in vitreoretinal surgery," Developments in Ophthalmology, vol. 42, pp. 115125, 2008.

[101] A. Ueno, T. Hisatomi, H. Enaida et al., "Biocompatibility of brilliant blue $\mathrm{G}$ in a rat model of subretinal injection," Retina, vol. 27, no. 4, pp. 499-504, 2007. 
[102] C. Creuzotgarcher, N. Acar, M. Passemard, S. Bidot, A. Bron, and L. Bretillon, "Functional and structural effect of intravitreal indocyanine green, triamcinolone acetonide, trypan blue, and brilliant blue G on rat retina," Retina, vol. 30 , no. 8 , pp. 1294-1301, 2010.

[103] S. Machida, T. Nishimura, T. Ohzeki, K. I. Murai, and D. Kurosaka, "Comparisons of focal macular electroretinograms after indocyanine green-, brilliant blue G-, or triamcinolone acetonide-assisted macular hole surgery," Graefes Archive for Clinical \& Experimental Ophthalmology, vol. 255, no. 3, pp. 485-492, 2017.

[104] K. Azuma, Y. Noda, K. Hirasawa, and T. Ueta, "Brilliant blue G-assisted internal limiting membrane peeling for macular hole: a systematic review of literature and meta-analysis," Retina, vol. 36, no. 5, pp. 851-858, 2016.

[105] A. Chandra, D. G. Charteris, and D. Yorston, "Posturing after macular hole surgery: a review," Ophthalmologica, vol. 226, Supplement 1, pp. 3-9, 2011.

[106] M. Alberti and C. M. La, "Nonsupine positioning in macular hole surgery: a noninferiority randomized clinical trial," Retina, vol. 36, no. 11, pp. 2072-2079, 2016.

[107] Z. Hu, P. Xie, Y. Ding, X. Zheng, D. Yuan, and Q. Liu, "Facedown or no face-down posturing following macular hole surgery: a meta-analysis," Acta Ophthalmologica, vol. 94, no. 4, pp. 326-333, 2016.

[108] R. W. Essex, Z. S. Kingston, M. Moreno-Betancur et al., "The effect of postoperative face-down positioning and of long- versus short-acting gas in macular hole surgery," Ophthalmology, vol. 123, no. 5, pp. 1129-1136, 2016.

[109] T. Xirou, P. G. Theodossiadis, M. Apostolopoulos et al., "Macular hole surgery with short-acting gas and shortduration face-down positioning," Clinical Ophthalmology, vol. 6, pp. 1107-1112, 2012.

[110] Y. Hasegawa, Y. Hata, Y. Mochizuki et al., "Equivalent tamponade by room air as compared with $\mathrm{SF}_{6}$ after macular hole surgery," Graefe's Archive for Clinical and Experimental Ophthalmology, vol. 247, no. 11, pp. 1455-1459, 2009.

[111] H. Usui, T. Yasukawa, Y. Hirano, H. Morita, M. Yoshida, and Y. Ogura, "Comparative study of the effects of room air and sulfur hexafluoride gas tamponade on functional and morphological recovery after macular hole surgery: a retrospective study," Ophthalmic Research, vol. 50, no. 4, pp. 227-230, 2013.

[112] S. S. Kim, W. E. Smiddy, W. J. Feuer, and W. Shi, “Outcomes of sulfur hexafluoride (SF6) versus perfluoropropane (C3F8) gas tamponade for macular hole surgery," Retina, vol. 28, no. 10, pp. 1408-1415, 2008.

[113] S. Briand, E. Chalifoux, E. Tourville et al., "Prospective randomized trial: outcomes of $\mathrm{SF}_{6}$ versus $\mathrm{C}_{3} \mathrm{~F}_{8}$ in macular hole surgery," Canadian Journal of Ophthalmology, vol. 50, no. 2, pp. 95-100, 2015.

[114] A. Modi, A. Giridhar, and M. Gopalakrishnan, "Sulfurhexafluoride (sf6) versus perfluoropropane (c3f8) gas as tamponade in macular hole surgery," Retina, vol. 37, no. 2, pp. 283-290, 2017.

[115] N. B. Kannan, O. O. Adenuga, K. Kumar, and K. Ramasamy, "Outcome of $2 \mathrm{cc}$ pure sulfur hexafluoride gas tamponade for macular hole surgery,” BMC Ophthalmology, vol. 16, no. 1, p. $73,2016$.
[116] L. Wagenfeld, O. Zeitz, C. Skevas, and G. Richard, "Long-lasting endotamponades in vitreoretinal surgery," Ophthalmologica, vol. 224, no. 5, pp. 291-300, 2010.

[117] X. Valldeperas and J. Lorenzo-Carrero, "Vitreous tamponades in highly myopic eyes," BioMed Research International, vol. 2014, Article ID 420380, 7 pages, 2014.

[118] V. Romano, F. Semeraro, M. R. Romano, M. Cruciani, and C. Costagliola, "Development of ocular hypertension secondary to tamponade with light versus heavy silicone oil: a systematic review," Indian Journal of Ophthalmology, vol. 63, no. 3, pp. 227-232, 2015.

[119] T. Avitabile, V. Bonfiglio, D. Buccoliero et al., "Heavy versus standard silicone oil in the management of retinal detachment with macular hole in myopic eyes," Retina, vol. 31, no. 3, pp. 540-546, 2011.

[120] H. Heimann, T. Stappler, and D. Wong, "Heavy tamponade 1: a review of indications, use, and complications," Eye, vol. 22, no. 10, pp. 1342-1359, 2008.

[121] B. Ivanovska-adjievska, S. Boskurt, F. Semiz, H. Yuzer, and V. Dimovska-Jordanova, "Treatment of idiopathic macular hole with silicone oil tamponade," Clinical Ophthalmology, vol. 6, pp. 1449-1454, 2012.

[122] H. Heimann, "Alternative indications for the use of heavy silicone oil tamponades," Klinische Monatsbltter Für Augenheilkunde, vol. 226, no. 9, pp. 713-717, 2009.

[123] J. C. Lai, S. S. Stinnett, and B. W. Mccuen, "Comparison of silicone oil versus gas tamponade in the treatment of idiopathic full-thickness macular hole," Ophthalmology, vol. 110, no. 6, pp. 1170-1174, 2003.

[124] H. H. Ghoraba, A. F. Ellakwa, and A. A. Ghali, "Long term result of silicone oil versus gas tamponade in the treatment of traumatic macular holes," Clinical Ophthalmology, vol. 6, pp. 49-53, 2012.

[125] M. E. Tafoya, H. M. Lambert, L. Vu, and M. Ding, "Visual outcomes of silicone oil versus gas tamponade for macular hole surgery," Seminars in Ophthalmology, vol. 18, no. 3, pp. 127-131, 2003.

[126] S. S. Couvillion, W. E. Smiddy, H. W. Flynn Jr, C. W. Eifrig, and G. Gregori, "Outcomes of surgery for idiopathic macular hole: a case-control study comparing silicone oil with gas tamponade," Ophthalmic Surgery, Lasers \& Imaging Retina, vol. 36, no. 5, pp. 365-371, 2006.

[127] M. Chakrabarti, P. Benjamin, K. Chakrabarti, and A. Chakrabarti, "Closing macular holes with "macular plug" without gas tamponade and postoperative posturing," Retina, vol. 37, no. 3, pp. 451-459, 2016.

[128] K. Masuyama, K. Yamakiri, N. Arimura, Y. Sonoda, N. Doi, and T. Sakamoto, "Posturing time after macular hole surgery modified by optical coherence tomography images: a pilot study," American Journal of Ophthalmology, vol. 147, no. 3, pp. 481-488.e2, 2009.

[129] D. Gupta, "Face-down posturing after macular hole surgery: a review," Retina, vol. 29, no. 4, pp. 430-443, 2009.

[130] C. Eckardt, T. Eckert, U. Eckardt, U. Porkert, and C. Gesser, "Macular hole surgery with air tamponade and optical coherence tomography-based duration of face-down positioning," Retina, vol. 28, no. 8, pp. 1087-1096, 2008.

[131] R. A. Mittra, J. E. Kim, D. P. Han, and J. S. Pollack, "Sustained postoperative face-down positioning is unnecessary for successful macular hole surgery," The British Journal of Ophthalmology, vol. 93, no. 5, pp. 664-666, 2009. 
[132] A. Tatham and S. Banerjee, "Face-down posturing after macular hole surgery: a meta-analysis," British Journal of Ophthalmology, vol. 94, no. 5, pp. 626-631, 2010.

[133] R. Iezzi and K. G. Kapoor, "No face-down positioning and broad internal limiting membrane peeling in the surgical repair of idiopathic macular holes," Ophthalmology, vol. 120, no. 10, pp. 1998-2003, 2013. 


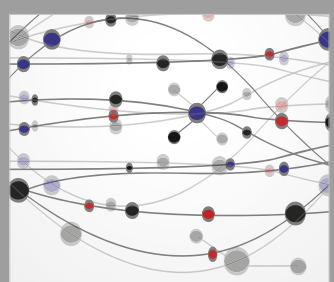

The Scientific World Journal
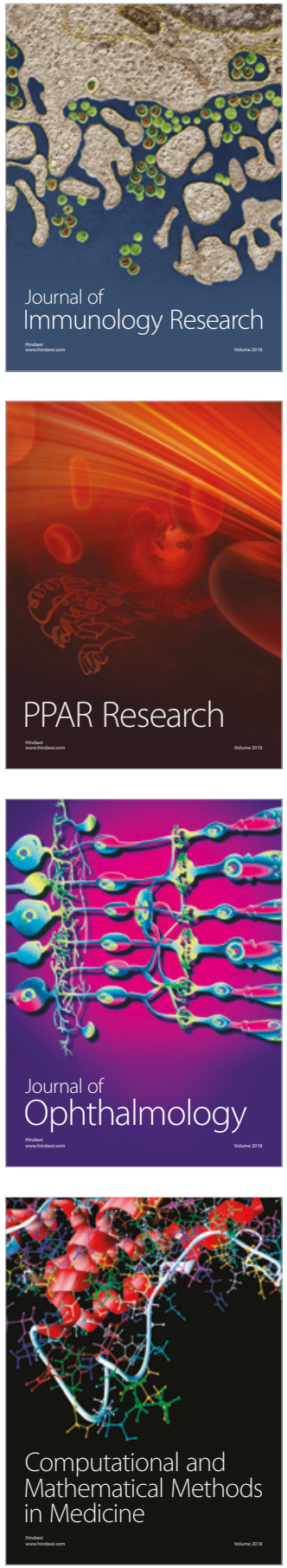

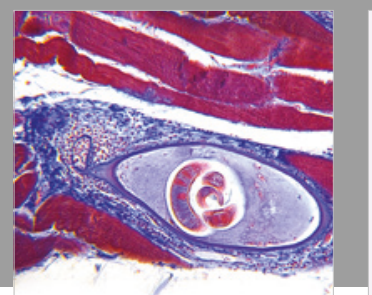

Gastroenterology Research and Practice

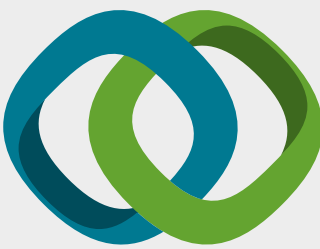

\section{Hindawi}

Submit your manuscripts at

www.hindawi.com
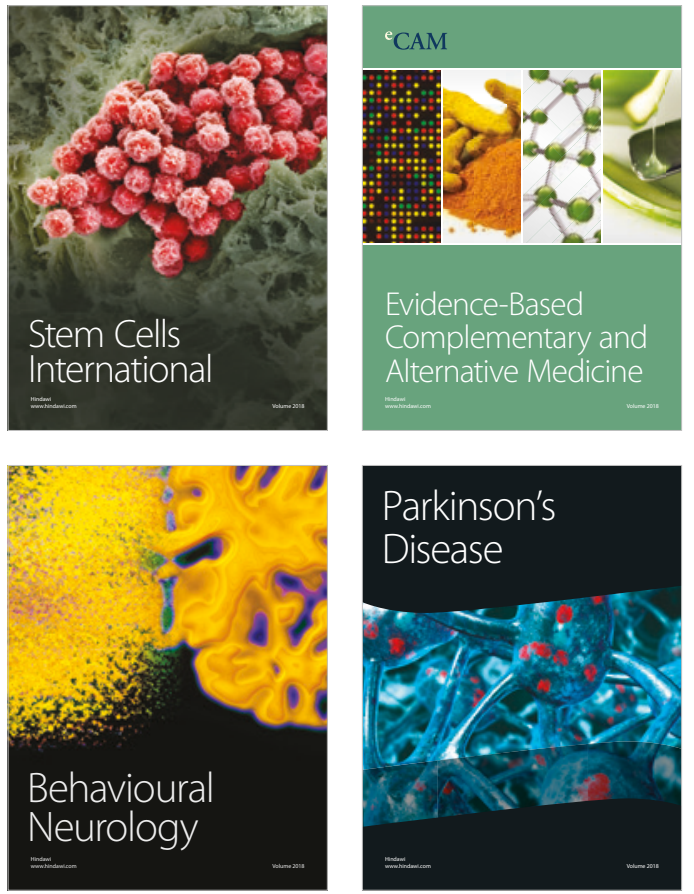

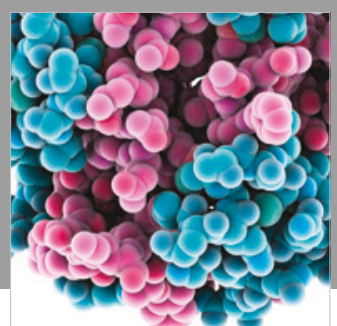

ournal of

Diabetes Research

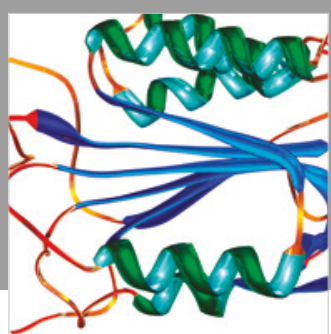

Disease Markers
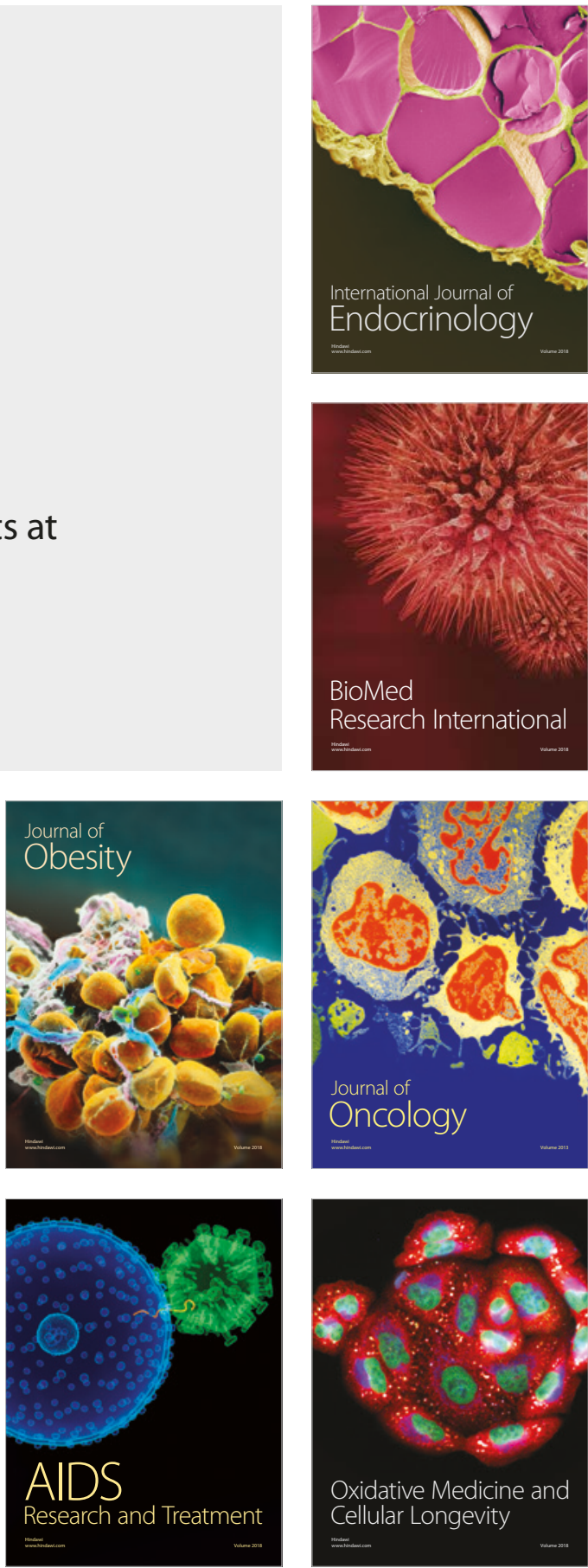